\title{
Ultrastructure, Histochemistry, and Mineralization Patterns in the Ecdysial Suture of the Blue Crab, Callinectes sapidus
}

\author{
C. Priester, R.M. Dillaman, D.M. Gay \\ University of North Carolina at Wilmington, Biological Sciences, Wilmington, NC, 28403-5915
}

The ecdysial suture is the predetermined region on an arthropod exoskeleton that splits open to allow the animal to escape from its old carapace [1]. In order to understand why this region preferentially splits, we examined the morphology and composition of the ecdysial suture of Callinectes sapidus using scanning electron microscopy (SEM) and light microscope histology and histochemistry.

No structural or compositional differences could be detected between the suture and the adjacent cuticle when stained with acridine orange (Fig. a) or hematoxylin and eosin. The suture region (in the center of the micrograph) possesses the same cuticular layers, epicuticle (arrowhead), exocuticle (ex), endocuticle (en) and membranous layer (arrow), as the adjacent cuticle with no apparent differences in thickness or affinity for these dyes. Staining with a battery of FITC-labeled lectins [2] revealed that only three were able to differentiate the suture. Lens culinaris agglutinin (Fig. b), Vicia faba agglutinin, and Pisum sativum agglutinin all bound intensely to the exocuticle in the region of the suture (arrowhead) and bound less to a wedge-shaped region of the endocutilcle (arrow). All three of these lectins have an affinity for fucosylated $\alpha \mathrm{N}$-acetylglucosamine with mannose dendrimers $[3,4]$.

SEM of the fracture surface of the cuticle of an intermolt crab in the region of the suture (Fig. c) shows that in the exocuticle of the suture the prisms are not in filled with calcium (arrowhead), whereas non-suture exocuticle is solid (arrow). Back-scattered electron analysis of a premolt cuticle embedded in resin, cut and polished (Fig. d) indicates that both the exocuticle (arrowhead) and the wedge-shaped endocuticle regions (arrow) of the suture are less dense than the surrounding cuticle. Non-dispersive X-ray microanalyses (Fig. e, f) clearly demonstrated that the suture is less mineralized than the surrounding cuticle and furthermore, has a significantly lower magnesium and phosphorus.

It appears, therefore, that the suture region of the blue crab, Callinectes sapidus, is different from the adjacent cuticle both in the composition of the organic matrix and the degree of mineralization. These characteristics appear to make the suture region more susceptible to the molting fluid [1] produced by the crab and therefore make it predetermined to fail when stressed by the internal pressured exerted by the new cuticle formed under the old cuticle or exuvia [5].

\section{References}

[1] P. Compère et al., Tissue and Cell (1998) 30: 41-56.

[2] R. Marlowe et al., J. Crustacean Biol. (1994) 14: 231-246.

[3] N.M. Young et al., Glycoconj. J. (1996) 13: 575-583.

[4] H. Debray et al. Eur. J. Biochem. (1981) 117: 41-55.

[5] This research was supported by NSF Grant number IBN 0114597 to RMD and a Sigma Xi Grant in Aid to Research to C. P. 
a

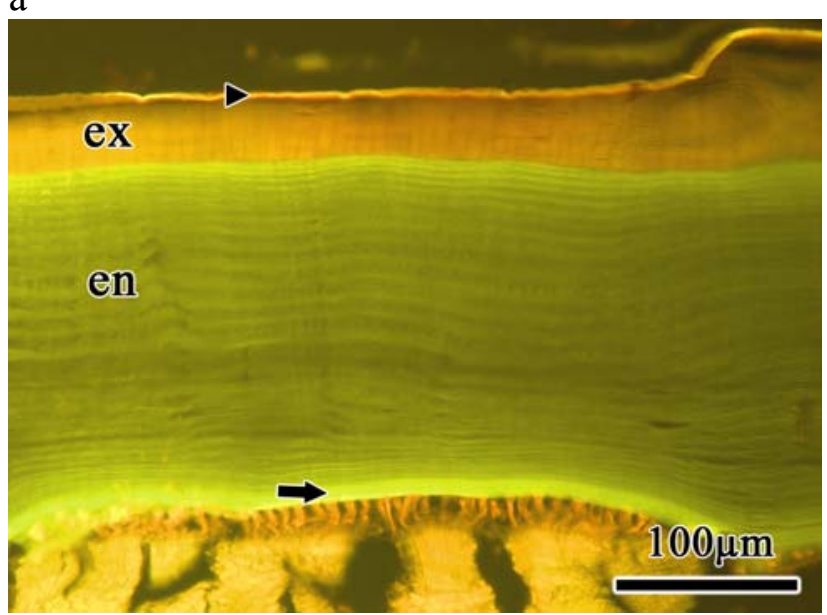

c

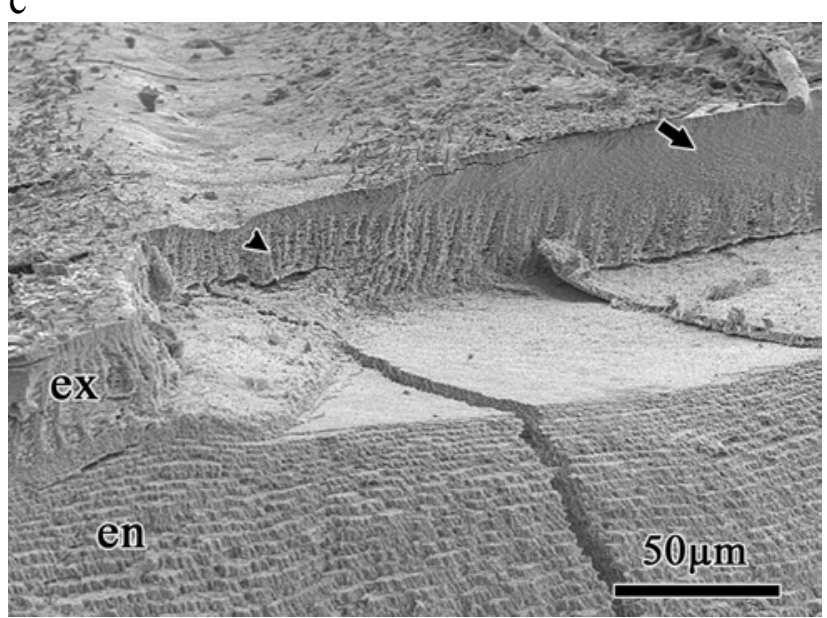

e

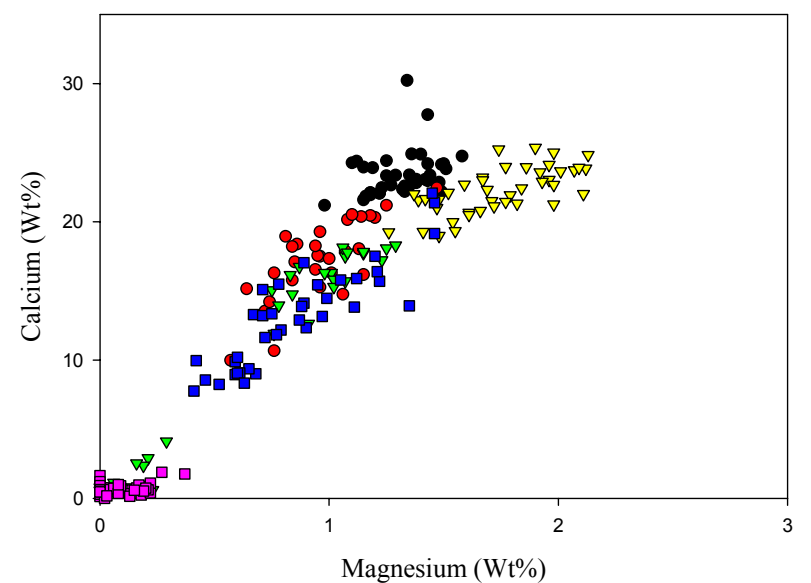

b

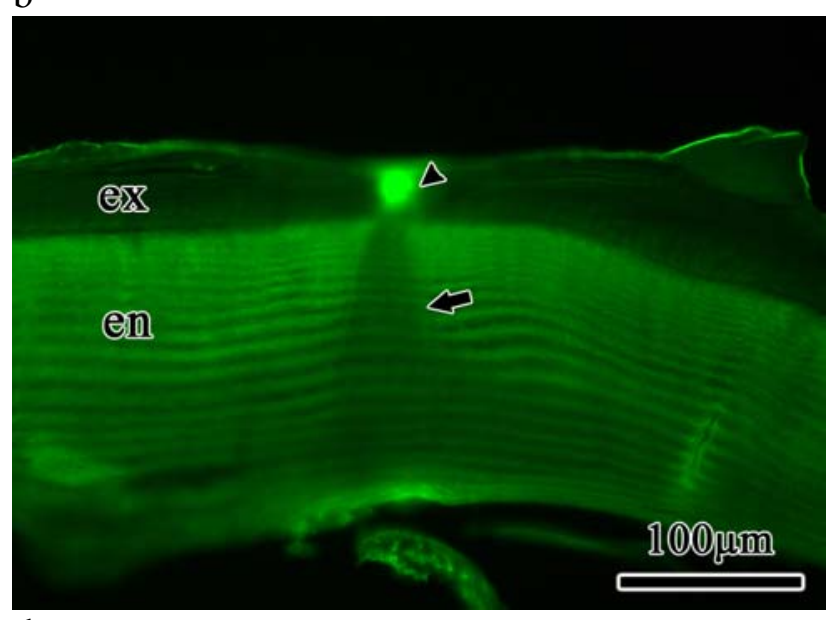

d

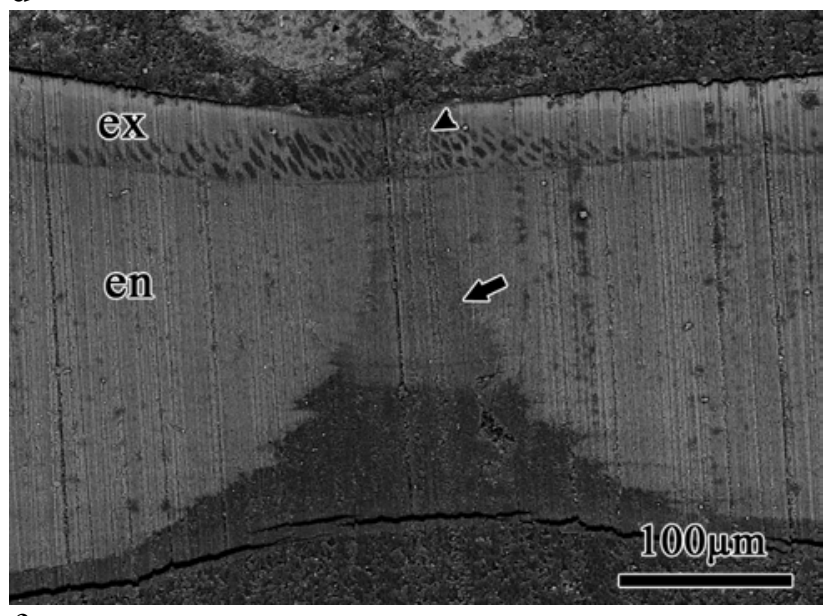

f

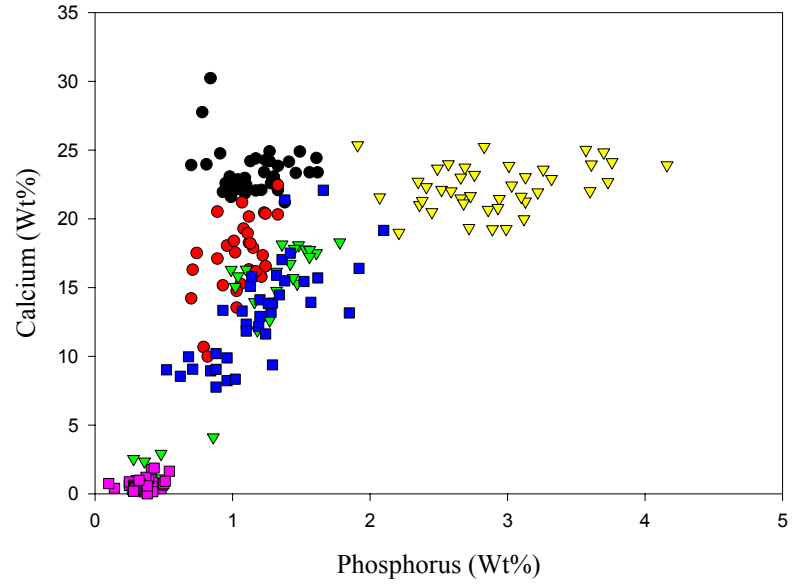

- endocuticle non-suture

- endocuticle suture (upper)

$\nabla \quad$ endocuticle suture (lower)

$\nabla \quad$ exocuticle non-suture

- exocuticle suture

- $\operatorname{resin}($ control) 